\section{Apolipoprotein E}

John DeLuca

Neuropsychology and Neuroscience Laboratory, Kessler Foundation Research Center, West Orange, NJ, USA

\section{Definition}

Apolipoprotein E (ApoE) is a polymorphic plasma glycoprotein that transports cholesterol and other lipids and has been shown to be involved in the growth and repair of neurons. There is also some evidence to suggest that ApoE is involved in lipid redistribution after demyelination. The ApoE protein is mapped to chromosome 19 and is polymorphic with three major isoforms, each of which translates into three alleles of the gene: ApoE-2, ApoE-3, and ApoE-4. ApoE-2 is associated with the genetic disorder type III hyperlipoproteinemia. There is also some evidence that this allele may serve as a protective role in the development of Alzheimer's disease (AD). ApoE-3 is found in approximately $64 \%$ of the population and is considered as the "neutral" ApoE genotype. ApoE-4 has been implicated in atherosclerosis and $\mathrm{AD}$ and impaired cognitive functioning. More specifically, ApoE-4 has been shown to be a major risk factor for development of $\mathrm{AD}$ and has been associated with subtle neuropsychological deficits in preclinical AD. Brain changes associated with ApoE-4 in $\mathrm{AD}$ include increased counts of amyloid plaques and neurofibrillary tangles, smaller medial temporal lobe structures, reduced glucose metabolism, and depletion of cholinergic markers in the hippocampus, frontal, and temporal cortices. ApoE-4 has also been associated with adverse recovery after traumatic brain injury (TBI). Persons with TBI with the ApoE-4 allele are ten times more likely to develop $\mathrm{AD}$ than those without the ApoE-4 allele. In multiple sclerosis, ApoE-4 has been found to be associated with rapid disease progression and increased cognitive impairment, although the findings for cognitive impairment have been inconsistent.

\section{Cross-References}

- Alzheimer's Disease

\section{References and Readings}

Plomin, R., Defries, J. C., Craig, I. W., \& McGuffin, P. (2003). Behavioral genetics in the postgenomic era. Washington, DC: American Psychological Association. 